\title{
THE MAGELLANIC STREAM
}

\author{
D. S. MATHEWSON and M. N. CLEARY \\ Mount Stromlo and Siding Spring Observatory, Australian National University, \\ Canberra, Australia \\ and \\ J. D. MURRAY \\ Division of Radiophysics, CSIRO, Sydney, Australia
}

\begin{abstract}
A southern sky survey of $\mathrm{HI}_{\mathrm{I}}$ in the velocity range $-340 \mathrm{~km} \mathrm{~s}^{-1}$ to $+380 \mathrm{~km} \mathrm{~s}^{-1}$ has shown that a long filament of $\mathrm{H} \mathrm{I}$ extends from the Small Magellanic Cloud (SMC) region down to the South Galactic Pole and connects with the long Hi filament discovered recently by Wannier and Wrixon (1972) and van Kuilenberg (1972). There is also some evidence that this continues on the other side of the Magellanic Clouds and crosses the galactic plane at $l=306^{\circ}$. This filament, which follows very closely a great circle over its entire $180^{\circ}$ arc across the sky, is given the name 'The Magellanic Stream'. It may have been produced by gravitational interaction between the SMC and the Galaxy during a close passage $(20 \mathrm{kpc})$ of the SMC some $5 \times 10^{8} \mathrm{yr}$ ago, although it is impossible to account for the observed radial velocities along the Stream unless some force other than gravity is invoked to act on the Stream as well.
\end{abstract}

Recently a southern sky survey has been made for $\mathrm{H}_{\mathrm{I}}$ in the velocity range $-340 \mathrm{~km} \mathrm{~s}^{-1}$ to $380 \mathrm{~km} \mathrm{~s}^{-1}$ using the $18-\mathrm{m}$ reflector at Parkes.

The main discovery of this survey is shown in Figure 1a, and that is the long filament of $\mathrm{HI}$ which extends from the region of the Small Magellanic Cloud (SMC) to the South Galactic Polar cap and beyond. This is given the name 'The Magellanic Stream'. All $\mathrm{H}_{\mathrm{I}}$ is plotted in this diagram except the 'zero-velocity' (i.e., local) gas. The average velocity half-width of the $\mathrm{HI}_{\mathrm{I}}$ in the Magellanic Stream is about $30 \mathrm{~km} \mathrm{~s}^{-1}$ which is much broader than the average half-width of $7 \mathrm{~km} \mathrm{~s}^{-1}$ of the local spiral arm gas.

Figure $1 \mathrm{~b}$ shows the areas of high positive velocity $\mathrm{HI}$ (greater than $200 \mathrm{~km} \mathrm{~s}^{-1}$ with respect to the local standard of rest and clearly resolved from spiral arm emission) discovered on the other side of the SMC and extending through the galactic plane at $l=306^{\circ}$ and up to $b=+30^{\circ}$. This gas may also belong to the Magellanic Stream. The two clouds centered on $l=289^{\circ}, b=20^{\circ}$, and $l=268^{\circ}, b=20^{\circ}$, were discovered by Wannier et al. (1972); the latter cloud has probably been produced by the expanding shell of the Gum nebula and should not be included in the Magellanic Stream.

Figure 2 shows the full extent of the Magellanic Stream on an Aitoff projection of the sky in galactic coordinates. The section between $l=320^{\circ}, b=-80^{\circ}$, and $l=80^{\circ}$, $b=-65^{\circ}$, had been surveyed previously by van Kuilenburg (1972); also see Dieter (1965) and Hulsbosch and Raimond (1966). Wannier and Wrixon (1972), using a more sensitive receiver, increased the observed length of this section of the Stream up to $l=90^{\circ}, b=-30^{\circ}$. The crosshatched patches are the clouds of high velocity $\mathrm{H}_{\mathrm{I}}$ discovered much earlier by northern hemisphere observers mostly at positive latitudes in the longitude range $80^{\circ}$ to $180^{\circ}$.

A striking feature of the Magellanic Stream is that it follows closely a great circle over its entire $180^{\circ}$ across the sky. If all the $\mathrm{H}_{\mathrm{I}}$ in the Stream were at the distance of the 


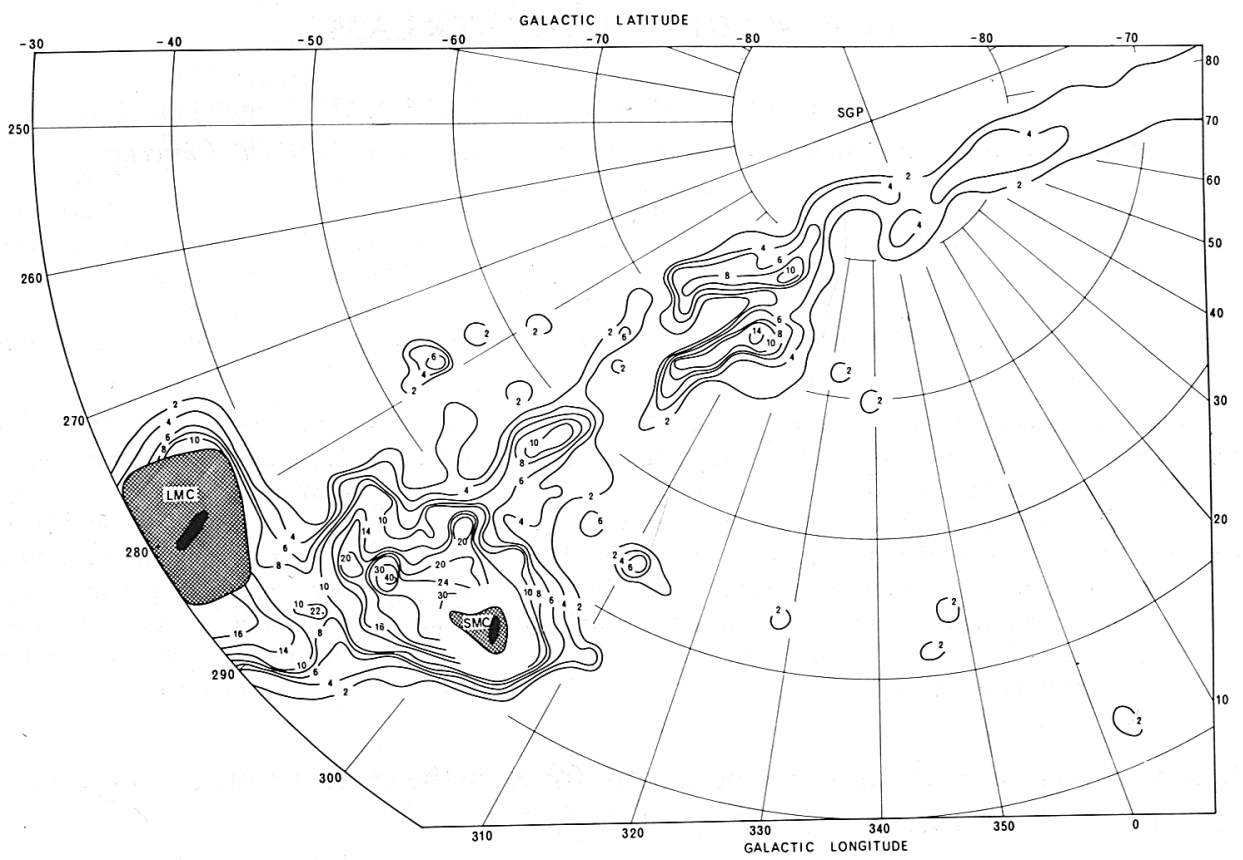

Fig. 1a. The section between the Magellanic Clouds and the South Galactic Polar region; all H I within the velocity range $-340 \mathrm{~km} \mathrm{~s}^{-1}$ to $+380 \mathrm{~km} \mathrm{~s}^{-1}$ is plotted except the 'zero-velocity' $\mathrm{H}$ i i.e., local spiral arm gas. The contours give the surface densities of the $\mathrm{H}_{\mathrm{I}}$ in the Magellanic Stream obtained using the $18-\mathrm{m}$ reflector at Parkes. The contour unit is $2 \times 10^{19}$ atoms $\mathrm{cm}^{-2}$. The cross-hatched regions represent the approximate optical extent of the Large Magellanic Cloud (LMC) and Small Magellanic Cloud (SMC).

SMC (63 kpc), its mass would be about $10^{9} \mathscr{M}_{\odot}$, which is equal to the combined mass of $\mathrm{HI}$ in the LMC and SMC. The gas mass of the inter-Cloud region between the SMC and the LMC accounts for half of this total.

Figure 3 shows the variation of radial velocity $\left(V_{\mathrm{GSR}}\right)$ with angular distance $(\theta)$ along the Stream using the coordinate system of Wannier and Wrixon (1972) in their Figure 2. The radial velocities referred to the local standard of rest (LSR) have been corrected for the galactic rotation at the Sun. These corrected velocities $\left(V_{\mathrm{GSR}}=\right.$ $=V_{\mathrm{LSR}}+225 \sin l \cos b$ ) are essentially velocities with respect to a non-rotating Galaxy. The systematic variation of radial velocity found by Wannier and Wrixon is seen to continue until the Magellanic Clouds are reached. However, on the other side of the Clouds there is no systematic variation of radial velocity, which produces a nagging doubt as to whether this $\mathrm{HI}_{\mathrm{I}}$ is really part of the Stream.

Many interpretations of the Magellanic Stream have been considered, but the most favoured one is that gravitational interaction between the SMC and the Galaxy has pulled out the Magellanic Stream from the SMC. Toomre (1973) in unpublished work (referred to by Mirabel and Turner, 1973) has constructed an orbit for the SMC 


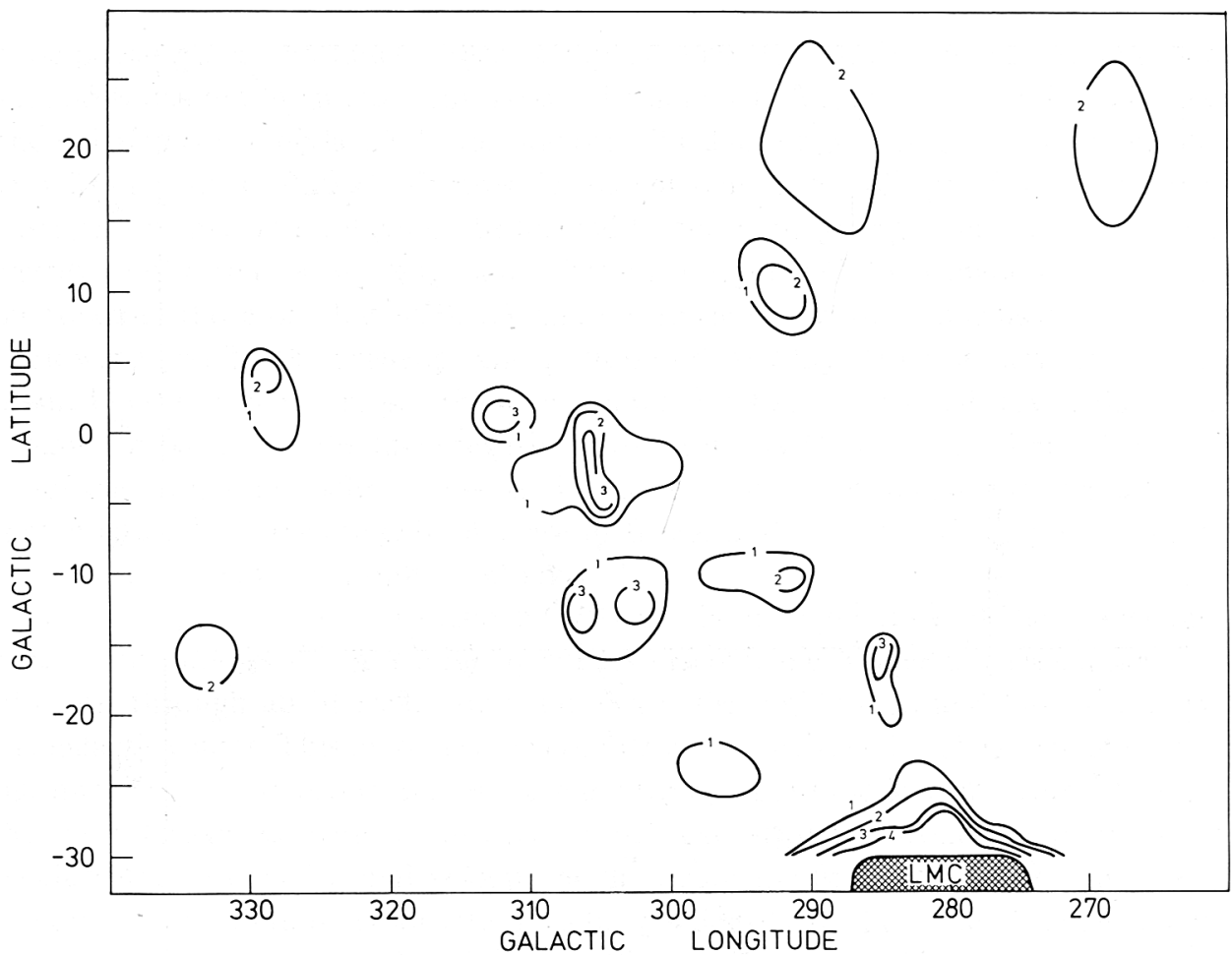

Fig. $1 \mathrm{~b}$. The section between the Magellanic Clouds and $+30^{\circ}$ galactic latitude; all $\mathrm{H}_{\mathrm{I}}$ greater than $+200 \mathrm{~km} \mathrm{~s}^{-1}$ relative to the local standard of rest is plotted. The two clouds centered on $l=289^{\circ}, b=20^{\circ}$, and $l=268^{\circ}, b=20^{\circ}$, were taken from Wannier et al. (1972). The latter cloud has probably been produced

by the expanding shell of the Gum nebula and should not be included in the Magellanic Stream.

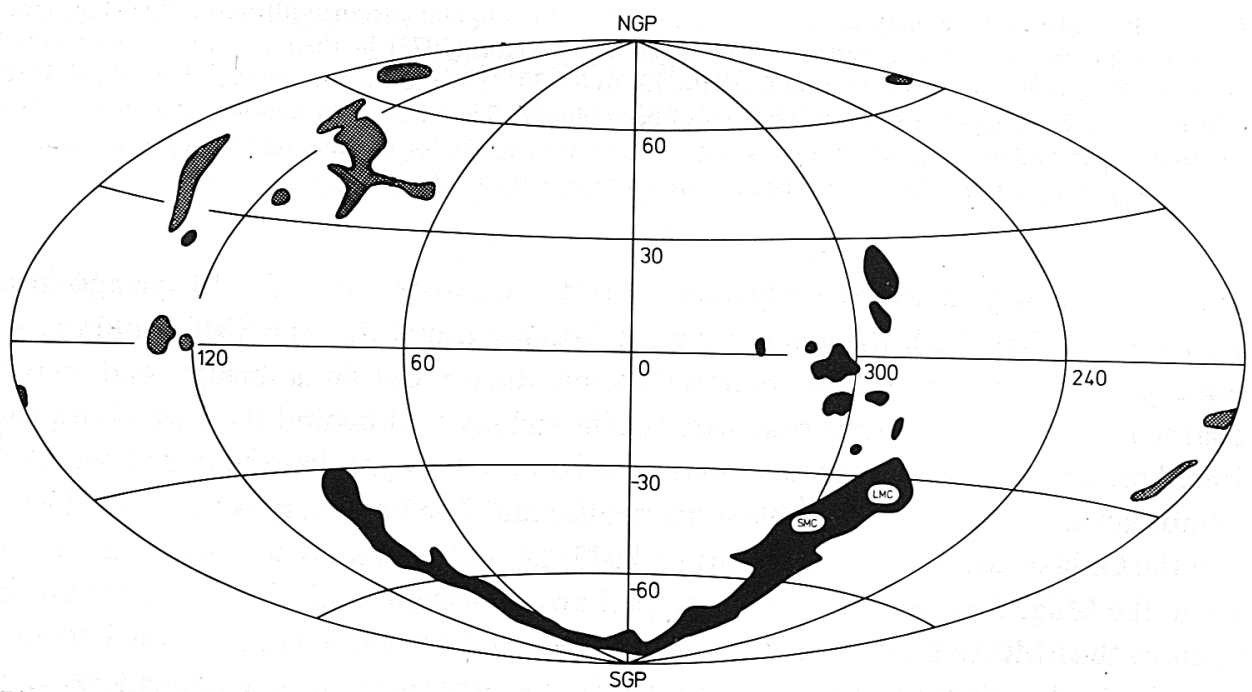

Fig. 2. The Magellanic Stream is drawn on this Aitoff projection in galactic co-ordinates. The crosshatched areas are the high velocity $\mathrm{H}$ I clouds discovered much earlier by northern hemisphere observers (cf. Hulsbosch, 1972). 


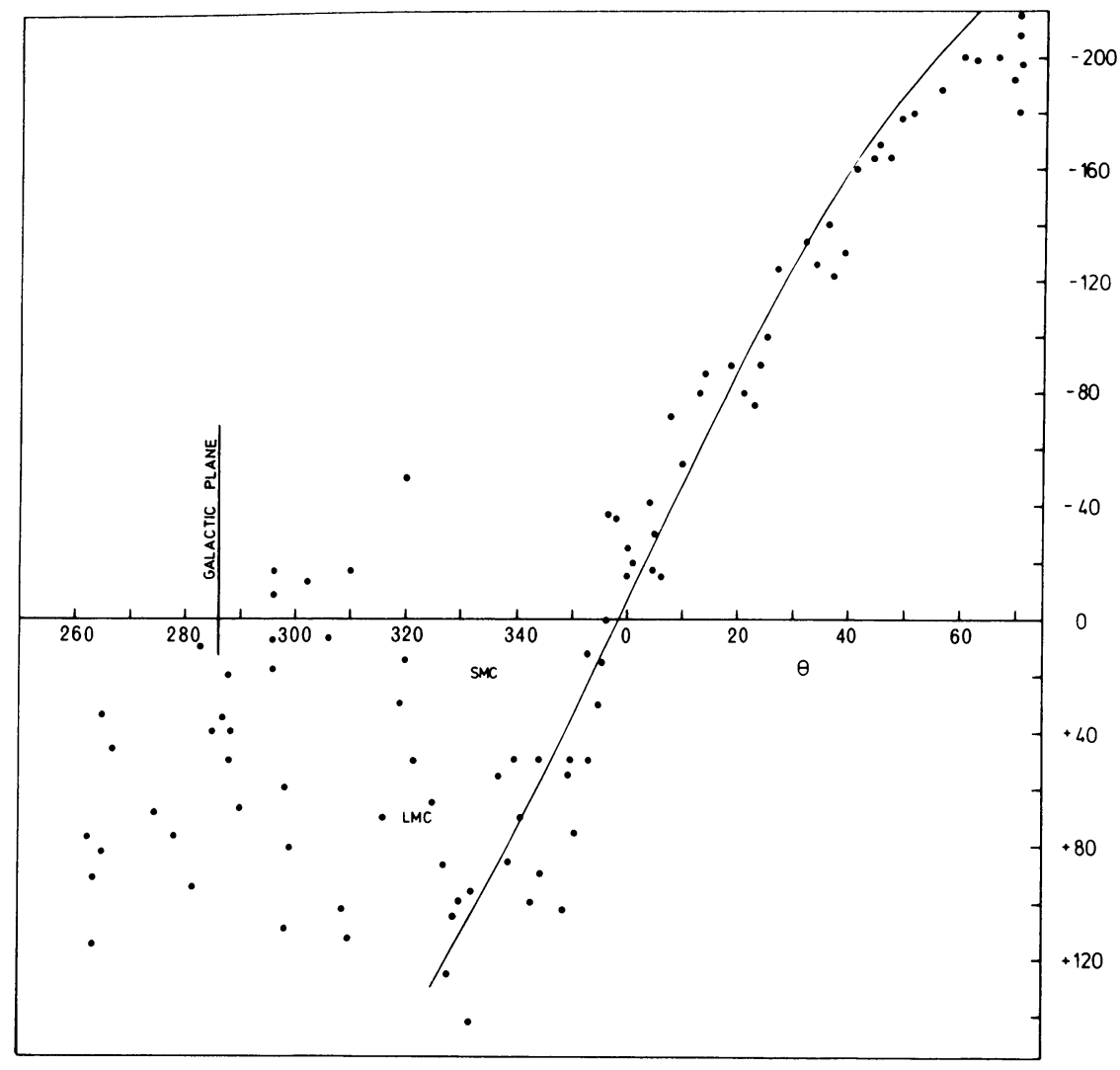

Fig. 3. Plot of radial velocity in $\mathrm{km} \mathrm{s}^{-1}\left(V_{\mathrm{GSR}}\right)$ versus the angular distance $(\theta)$ along the Magellanic Stream using the co-ordinate system of Wannier and Wrixon (1972) in their Figure 2. Their radial velocities have been used in the section from $\theta=70^{\circ}$ to $\theta=30^{\circ}$. The systemic radial velocities of the SMC $\left(20 \mathrm{~km}^{-1} \mathrm{~s}\right)$ and the LMC $\left(68 \mathrm{~km} \mathrm{~s}^{-1}\right)$ have also been plotted. The radial velocities $\left(V_{\mathrm{GSR}}\right)$ refer to a nonrotating galaxy and are obtained from the velocities relative to the local standard of rest by the relation $V_{\mathrm{GSR}}=V_{\mathrm{LSR}}+225 \sin l \cos b$. The full line represents the relation $V_{\mathrm{GSR}}=-240 \sin \left(\theta+2^{\circ}\right)$.

about the Galaxy in which perigalactic passage occurred some $5 \times 10^{8}$ yr ago at a distance of $20 \mathrm{kpc}$ from the galactic center. Toomre found that the SMC would have undergone severe disruption, material being drawn out in a bridge and a tail characteristic of such violent tidal events. The tail lay well behind the LMC but projected onto the line joining the two galaxies. Toomre thought that the inter-Cloud $\mathrm{H}_{\mathrm{I}}$ (Hindman et al., 1963; Turner, 1968) may be the tail. The bridge lay between the SMC and the Galaxy (see Mirabel and Turner, 1973) but unfortunately was angled at about $40^{\circ}$ to the Magellanic Stream. However, perhaps on reexamination, it may be possible to allow the SMC to have a more highly inclined orbit about the Galaxy, which would shift the bridge closer to the South Galactic Pole and more nearly coincident with the Magellanic Stream.

Clutton-Brock (1972) has produced plots to show the tearing of a galaxy by the 
close passage of a very much larger galaxy. He finds that under these conditions, the gas and stars are drawn out from the smaller galaxy to form a very prominent bridge and tail. If the orbit of the SMC was highly inclined to the galactic plane then it may be seen from Clutton-Brock's Figures $4 \mathrm{~g}$ and $4 \mathrm{i}$ that in $5 \times 10^{8} \mathrm{yr}$ after perigalactic passage, a bridge and tail would be formed by the SMC giving a very similar spatial distribution to that of the Magellanic Stream. Wright (1973) has made similar computations to those of Clutton-Brock and has reached a similar conclusion.

However, whilst the existence and position of the Magellanic Stream may be explained in this way, it is impossible to explain the radial velocities observed along the Stream. In particular the high negative radial velocity $\left(V_{\mathrm{GSR}}=-216 \mathrm{~km} \mathrm{~s}^{-1}\right)$ at the tip of the Stream at $l=90^{\circ}, b=-30^{\circ}$, is almost an order of magnitude greater than that predicted by the models of Clutton-Brock and Wright, which give a radial velocity in this region of only $-30 \mathrm{~km} \mathrm{~s}^{-1}$.

Oort (1973), who worked independently on the interpretation of the Magellanic Stream, overcomes this difficulty by suggesting that the Magellanic Stream 'snowploughs' through an intergalactic wind and is thereby braked and blown towards the galactic center. This increases the component of the velocity of the Stream along our line of sight and also increases the velocity of infall of the Stream. Oort calculates that an intergalactic wind of density $2 \times 10^{-4}$ atoms $\mathrm{cm}^{-3}$ would be sufficient to produce the observed radial velocity at the tip of the Stream. This seems quite a reasonable speculation, as an intergalactic gas density of this order is necessary to explain the stability of the Local System (Oort, 1970). In addition Oort invokes an intergalactic wind of this density to explain the high velocity $\mathrm{H}_{\mathrm{I}}$ clouds found by northern hemisphere observers at positive latitudes between longitudes $80^{\circ}$ and $180^{\circ}$.

However, there are difficulties with this concept because denser parts of the Stream such as that around $l=300^{\circ}, b=-73^{\circ}$, would be relatively unaffected by snowploughing through the intergalactic wind and would continue along their original orbits so that they should have more positive radial velocities than the nearby less dense sections. However, the reverse is the case. In addition it is difficult to explain the positive radial velocities in the Stream near the Magellanic Clouds(e.g., $130 \mathrm{~km} \mathrm{~s}^{-1}$ at $l=286^{\circ}, b=-47^{\circ}$, and $90 \mathrm{~km} \mathrm{~s}^{-1}$ at $\left.l=294^{\circ}, b=-58^{\circ}\right)$ compared to the systemic radial velocities of $20 \mathrm{~km} \mathrm{~s}^{-1}$ for the SMC and $68 \mathrm{~km} \mathrm{~s}^{-1}$ for the LMC.

It is tempting to speculate that part of the Magellanic Stream may already have hit the galactic plane within $5 \mathrm{kpc}$ of the Sun at $l \approx 100^{\circ}$ and that its momentum pushed some disk gas to $z$ distances of several kiloparsecs. As the width of the Stream is about 5 to $10 \mathrm{kpc}$, the disk gas would have been disturbed over quite a broad range of galactic longitudes around $l=100^{\circ}$. At this point the mechanism put forward by Oort (1970) to explain the origin of the high velocity $\mathrm{H}$ I clouds (cross-hatched in Figure 2) may operate. That is that the gas falls back into the galactic plane under the pressure of the intergalactic wind and the gravitational field of the Galaxy. This action of the Magellanic Stream on the $\mathrm{H}_{\mathrm{I}}$ in the plane replaces the need for super-explosions postulated by Oort (1970) to replenish the gas in the halo.

This interesting discovery has many follow-up observations, the most important of 
which is to search for optical emission. In this regard Bok (1966) commented that a striking feature of the distribution of globular clusters in the Clouds is that many of them are far from the two galaxies and indeed some of them cannot be assigned membership specifically to either Cloud because of this (Gascoigne and Lyngå, 1963).

This 'discovery' paper would not be complete without mentioning some earlier papers which, although not necessarily correct, were 'hot on the trail' of the Magellanic Stream. Nearly twenty years ago de Vaucouleurs (1954) discussed the possibility of a connection between the Magellanic Clouds and the Galaxy and coined the name 'The Magellanic Stream'; and Kerr and Sullivan (1969) considered that the high velocity $\mathrm{H}$ I clouds known at that time may be satellites of the Galaxy (perhaps debris scattered around the orbit of the LMC) at distances of the order of $50 \mathrm{kpc}$. It should also be noted that Hulsbosch (1972) when discussing the long South Polar filament found by van Kuilenburg and Wannier and Wrixon, suggested that "it may be a tidal arm expelled from the LMC by an encounter with the Galaxy".

\section{Acknowledgements}

The authors acknowledge valuable discussions about the interpretation of this feature with Prof. Toomre, Dr Kalnajs and Dr Hulsbosch, and they thank Prof. Oort for communicating the results of his work on the interpretation of the Magellanic Stream prior to their publication. The authors would also like to thank David Cooke, Frank Trett and Bob Phelps of the resident engineering staff at Parkes for keeping the receiver operational and Gary da Costa and Graham White from Mt. Stromlo for assistance with the observations.

\section{References}

Bok, B. J.: 1966, Ann. Rev. Astron. Astrophys. 4, 95.

Clutton-Brock, M.: 1972, Astrophys. Space Sci. 17, 292.

Dieter, N. H.: 1965, Astron. J. 70, 552.

Gascoigne, S. C. B. and Lyngå, G.: 1963, Observatory 83, 38.

Hindman, J. V., Kerr, F. J., and McGee, R. X.: 1963, Australian J. Phys. 16, 570.

Hulsbosch, A. N. M.: 1972, Studies on High-Velocity Clouds, dissertation, Leiden University.

Hulsbosch, A. N. M. and Raimond, E.: 1966, Bull. Astron. Inst. Neth. 18, 413.

Kerr, F. J. and Sullivan, W. T., III : 1969, Astrophys. J. 158, 115.

Kuilenberg, J. van: 1972, Astron. Astrophys. 16, 276.

Mirabel, I. F. and Turner, K. C.: 1973, Astron. Astrophys. 22, 437.

Oort, J. H.: 1970, Astron. Astrophys. 7, 381.

Oort, J. H.: 1973, private communication.

Toomre, A.: 1973, private communication.

Turner, K. C.: 1968, Annual Report of the Director, Department of Terrestrial Magnetism 1967-68, Carnegie Inst. of Washington, p. 291.

Vaucouleurs, G. de: 1954, Observatory 74, 23.

Wannier, P. and Wrixon, G. T.: 1972, Astrophys. J. Letters 173, L119.

Wannier, P., Wrixon, G. T., and Wilson, R. W.: 1972, Astron. Astrophys. 18, 224.

Wright, A. E. : 1973, private communication. 
D. S. Mathewson

M. N. Cleary

Mt. Stromlo \& Siding Spring Observatory,

Private Bag,

Woden, A.C.T. 2606, Australia

\author{
J. D. Murray \\ CSIRO Division of Radiophysics, \\ P.O. Box 76, \\ Epping, N.S.W. 2121, Australia
}

\title{
DISCUSSION
}

Pishmish: How are the distances of the high velocity clouds determined?

Davies: Where the HVC is at lower latitudes and associated clearly with the outer spiral structure a dynamical distance may be estimated. Otherwise, the neutral hydrogen data by themselves do not give a distance (e.g., HVCs at high latitudes or in the anticenter).

Habing: Distances to high-velocity clouds can be obtained in principle by observing interstellar lines in distant stars. This method has so far not been successful although in a few cases distances of at least a kiloparsec been obtained.

Habing: What makes you think that those separate clouds belong to the Stream that lie on the extrapolation of the Stream beyond the Magellanic Clouds? Their velocities do not seem to indicate a relationship to the Stream, and their position beyond the Clouds makes, at first sight, their relation to the Stream doubtful.

Mathewson: The reasons why they have been included in the Stream are (1) they have very high positive velocities, which sets them apart from the spiral arm emission, (2) they lie close to the great circle followed by the Magellanic Stream on the other side of the Clouds, and (3) they are elongated in the direction of the Stream, which is at right angles to the galactic plane. However, the lack of systematic variations in the radial velocities is worrying and produces a doubt as to whether they do belong to the Stream, partiularly as the high positive velocity cloud at $l=268^{\circ}, b=20^{\circ}$, is almost certainly associated with the Gum nebula.

Oort: Davies has described the interpretation that Verschuur and he have suggested for the highvelocity clouds as parts of distant spiral arms bent away from the galactic plane. They have suggested also that the elongated structures shown by several of the high-velocity features connect up with gas close to the plane. The possibility of such a connection has recently been investigated in detail by Hulsbosch for the large high-velocity complex which has been called cloud $\mathrm{A}$. He found no evidence for such a connection. In my opinion the real nature of high-velocity 'clouds' or 'streams', can best be seen in those at high and intermediate latitudes. A characteristic of these streams is an extreme unevenness in density as well as velocity. In cloud $A$ one finds velocity jumps from one region to another of $40 \mathrm{~km} \mathrm{~s}^{-1}$ or more; in the so-called anticenter complex these differences run up to $100 \mathrm{~km} \mathrm{~s}^{-1}$. These high internal motions suggest that what we observe in these streams is an interaction between two streams of gas. This is also indicated by the sharp edges found in some of these objects. An interaction of such a nature could occur as a consequence of the collision of an infalling wind of inter-galactic gas with the highly irregular gaseous halo of the Galaxy.

Davies: I agree that the higher latitude HVCs will give important clues about the origin of HVCs. Since the lower latitude HVCs are closely associated with the outer spiral arms, I would argue that we should first look for an origin involving material in some way associated with this outer spiral structure. On this basis the known tidal interaction between the Milky Way and the Magellanic Clouds seems to offer a ready explanation in terms of material pulled from the plane in the outer regions of the Milky Way or indeed material actually torn out of the Magellanic Clouds. Both negative and positive velocities can be produced in this way, and furthermore the material is likely to be in bands having a substantial velocity dispersion.

Mathewson: I think probably it's more unified than that. The Magellanic Stream may actually cause the high velocity clouds of northern latitudes. The Stream is coming down on the galactic plane, and it can 
quite easily eject the disk material. It is coming in at $250 \mathrm{~km} \mathrm{~s}^{-1}$, and it has got a lot of mass. It can quite easily carry the gas in the disk afterwards. There is a bit of a gap here, but it only takes $10^{7} \mathrm{yr}$ to cover that gap. I think the clouds may have shot up 3-5 kpc. What the Northern Hemisphere observes is this gas falling back into the disk, probably pushed by the inter-galactic wind as Oort has suggested.

Van Woerden: The interpretation of most of the high-velocity clouds at high $b$ as halo gas pushed down by infalling intergalactic matter appears to be supported by:

(i) the presence of a large intermediate - negative - velocity (INV) complex at very high positive latitudes: $b \sim+70^{\circ}$ (Blaauw and Tolbert: 1966, Bull. Astron. Inst. Neth. 18, 405)

(ii) the velocity field of this complex, indicating a flow at $70 \mathrm{~km} \mathrm{~s}^{-1}$ coming from $l \sim 120, b \sim 40$ (Wessalius and Fejes: 1973, Astron. Astrophys. 24, 20, Fig. 3, and p. 33, Fig. 11);

(iii) the hole in the distribution of low-velocity hydrogen centered at $l \sim 160^{\circ}, b \sim+70^{\circ}$ (Wesselius and Fejes: 1973, Astron. Astrophys. 24, 25, Fig. 6) and coinciding approximately with the INV complex.

Guélin: Evidence of intergalactic gas of tenuous density has been brought in evidence in the multiple system M81-M82-NGC 3077 (R. Davies, IAU Symp. 58; M. Guélin, XVth General Assembly).

Pishmish: Has the search of the high velocity clouds been systematic? Are all galactic longitudes covered by the search?

Mathewson: Yes. 